\title{
Estimação de Tempo em Ratos Submetidos a um Procedimento de Discriminação Condicional
}

\author{
Rat's Time Estimation under Conditional Discrimination Procedure \\ José Lino Oliveira Bueno*, Alexandre Wagatsuma \& Mateus Russomanno Martins \\ Universidade de São Paulo, Ribeirão Preto, Brasil
}

\begin{abstract}
Resumo
Num procedimento de discriminação condicional (DC) de característica positiva, um mesmo estímulo alvo A não é seguido de reforço quando apresentado sozinho, mas é seguido de reforço quando precedido de um estímulo X. Na DC de característica negativa, um mesmo estímulo alvo B é seguido de reforço quando apresentado sozinho, mas nunca quando precedido do estímulo Y. Nesta pesquisa, procurou-se examinar se uma informação amodal relativa a atributos básicos de estímulos, como a duração, tem capacidade de adquirir propriedades condicionais em animais. Ratos foram submetidos a procedimentos de DC positiva e negativa com estímulos X e Y de mesma modalidade física, uma luz, mas com durações diferentes: $\mathrm{X}=15$-seg e $\mathrm{Y}=5$-seg; as durações dos estímulos alvo foram $\mathrm{A}=5$-seg e $\mathrm{B}=15-\mathrm{seg}$. Os resultados mostraram que os animais discriminaram as propriedades condicionais excitatórias (X) e inibitórias (Y) relativas, respectivamente, a duas durações diferentes de um mesmo estímulo, sustentando a hipótese de que a duração, um componente amodal de estímulo, pode ser associada a propriedades condicionais, em ratos.

Palavras-chave: Discriminação condicional; controle temporal; representação de estímulos; condicionamento Pavloviano.

Abstract

In a feature positive conditional discrimination procedure, a stimulus A is not followed by reinforcement when presented alone, but it is always followed by reinforcement when it is preceded by stimulus X. In the negative conditional discrimination procedure, a stimulus B is always followed by reinforcement when presented alone, but it is not followed by reinforcement when preceded by stimulus Y. This research examined if amodal information dealing with basic attributes, as duration, has the capability to acquire conditional properties in animals. Rats were submitted to positive or negative conditional discrimination procedures with stimulus $\mathrm{X}$ and $\mathrm{Y}$, which were of the same physical modality, a light, but with different durations: $\mathrm{X}=15 \mathrm{seg}$ e $\mathrm{Y}=5 \mathrm{seg}$ (and $\mathrm{A}=5 \mathrm{seg}, \mathrm{B}=15 \mathrm{seg}$ ). The results showed that the animals discriminated excitatory and inhibitory properties related, respectively, to two different durations of the same stimulus, supporting the hypothesis that the duration, as an amodal stimulus component, can be associated to conditional properties, in rats.

Keywords: Conditional discrimination; temporal control; stimuli representation; Pavlovian conditioning.
\end{abstract}

Vários estudos envolvendo condicionamento Pavloviano e operante, em procedimentos de discriminação condicional, têm sido utilizados com o objetivo de melhor se entender os processos envolvidos no estabelecimento das associações entre seus elementos primários: estímulo, resposta e reforço. Jenkins e Sainsbury (1969) descreveram um procedimento de discriminação condicional, no qual um composto de estímulos XA apresentado simultaneamente seguido de liberação de um estímulo incondicionado (US); entretanto, quando o estímulo A era apresentado sozinho não era seguido de estímulo incondicionado. Os resultados mostraram que, com o treino, os animais foram capazes de discriminar propriedades excitatórias e inibitórias de um mesmo estímulo A, dependendo da condição desse ter sido acompa-

\footnotetext{
* Endereço para correspondência: Departamento de Psicologia e Educação da Faculdade de Filosofia, Ciências e Letras, Universidade de São Paulo, Av. dos Bandeirantes, 3.900, Ribeirão Preto, SP, 14040-901. Fone/fax: (55) 16-36023697, Fax: (55) 16-3633-5668. E-mail: jldobuen@ffclrp.usp.com.br

Apoio financeiro: Pesquisador I CNPq; FAPESP.
} nhado ou não por um outro estímulo X. O estímulo X tem sido denominado por estímulo característica ou condicional e o estímulo $\mathrm{A}$, comum às duas práticas, tem sido denominado como estímulo alvo ou condicionado. Bueno e Moreira (1998) descreveram, num dos experimentos, um procedimento de discriminação condicional, com práticas nas quais eram apresentados sucessivamente Luz de Painel, um intervalo vazio (IV) e um Ruído Branco, com durações de 5 segundos para cada evento, seguidos da liberação de uma gota de água, como estímulo incondicionado (X IV A+); entretanto, em práticas em que o Ruído Branco era apresentado sozinho esse não era seguido de água (A-). Os resultados mostraram que, com o treino, os animais apresentaram freqüências maiores de respostas de orientação ao bebedouro durante o Ruído Branco precedido da Luz de Painel do que durante a apresentação do Ruído Branco sozinho. Assim, os animais foram capazes de discriminar proprieda- 
des excitatórias e inibitórias de um mesmo estímulo Ruído Branco (A), dependendo da condição esse ter sido precedido ou não por uma Luz de Painel (X).

Ross e Holland (1981) mostram que fatores temporais afetam o desempenho do animal em procedimentos de discriminação condicional. Tem sido demonstrado que o arranjo temporal, envolvendo o estímulo característica $(\mathrm{X})$ e o estímulo condicionado (A), é importante para a determinação das propriedades do estímulo característica. A apresentação dos estímulos XA pode ser simultânea ou seriada.

Num procedimento de discriminação condicional simultânea um estímulo composto com a apresentação simultânea dos seus elementos é seguido pelo estímulo incondicionado ou reforçador $(\mathrm{XA}+)$, mas um de seus elementos (A-) quando apresentado sozinho não é seguido pelo estímulo incondicionado. Supõe-se que o estímulo característica $\mathrm{X}$ age como um eliciador de resposta a um estímulo incondicionado, e que o estímulo A tem pouco ou nenhum controle sobre o comportamento. Assim, numa discriminação de característica positiva simultânea há uma associação condicionada primária entre o estímulo característica $(\mathrm{X})$ e o estímulo incondicionado (+). Num teste de transferência, o estímulo alvo empregado na fase de treino (A) é substituído por outro estímulo alvo (B). Se o animal foi treinado com o procedimento simultâneo, a propriedade do estímulo característica $(\mathrm{X})$ se transfere para outro estímulo (B) diferente do empregado no treino (A).

Em outra situação, num procedimento de discriminação seriada, os mesmos estímulos compostos são apresentados serialmente ao animal com um intervalo vazio - um intervalo sem a apresentação de um estímulo - entre os estímulos (práticas X IV A+ e A-). Nesse caso, supõe-se que o estímulo característica X tem uma função moduladora sobre o estímulo eliciador. Numa discriminação de característica positiva seriada o estímulo $\mathrm{X}$ define a ocasião (occasion setting) em que se dá associação primária entre o estímulo A e o estímulo incondicionado. Assim, num teste de transferência, a propriedade do estímulo característica $(\mathrm{X})$ adquirida com treino serial não se transfere para outro estímulo (B) diferente do empregado no treino (A).

Os testes de transferência, portanto, permitem a identificação do processo subjacente ao desempenho do animal em discriminação condicional. Se há transferência das propriedades do estímulo característica, o animal responderá durante a apresentação do estímulo alvo, que não fora treinado precedido deste estímulo característica, da mesma forma que durante o estímulo alvo que fora precedido, no treino, pelo estímulo característica, indicando que a aprendizagem envolveu uma associação primária entre o estímulo característica e o US. Entretanto, se não há transferência das propriedades do estímulo característica, o animal não responderá da mesma forma que durante o estímulo alvo que fora precedido no treino pelo estímulo característica, indicando que o estímulo característica tem a propriedade de definir a ocasião (occasion setting) da ocorrência da associação primária específica CS-US.

Vários pesquisadores (Holland, 1984, 1985; Pearce \& Hall, 1980; Rescorla \& Holland, 1977; Wagner \& Rescorla,
1972) têm mostrado interesse não apenas por propriedades excitatórias do estímulo característica em discriminações positivas, mas também esse interesse estende-se para suas propriedades inibitórias no procedimento de característica-negativa (Jenkins \& Sainsbury, 1969) ou inibição condicionada (Pavlov, 1927). Holland e Lamarre (1984), estudando este procedimento no qual um estímulo condicionado A é seguido de estímulo incondicionado (+) quando apresentado sozinho, mas não é seguido do estímulo incondicionado quando precedido pelo estímulo característica $\mathrm{X}$, verificaram a propriedade de $\mathrm{X}$ em inibir respostas condicionadas para A.

A modalidade do estímulo tem sido uma das variáveis estudadas em procedimentos de discriminação condicional. Há estudos mostrando que a topografia das respostas condicionadas é relacionada aos estímulos empregados, em procedimentos de discriminação condicional. Ross e Holland (1981) submeteram ratos a práticas com estímulos compostos luztom simultâneos (XA), seguidos pelo estímulo incondicionado comida, e a práticas com tom (A), não seguido do estímulo incondicionado; no mesmo experimento os ratos foram submetidos a procedimentos com estímulos compostos seriados (X IV A). Associações entre o estímulo característica visual e o US eram indexados pelo comportamento de levantar-se e associações entre o estímulo CS auditivo e o US eram indexadas pelo comportamento head-jerk + lamber e farejar o bebedouro. Os resultados mostraram que o estímulo auditivo ou estímulo condicionado evocou comportamentos condicionados específicos a estímulos auditivos - tais como head-jerk+ lamber e farejar o bebedouro - somente quando foi precedido pelo estímulo característica visual (X IV A).

Estudos com animais têm mostrado que componentes amodais de estímulos podem também adquirir propriedades discriminativas simples. Os estudos de Meck e Church (1982) estão orientados para procedimentos com testes intermodais (cross-modal) para demonstrar que a informação de estimulo sobre atributos básicos de estímulos, tais como duração, pode ser idêntica quando as modalidades de estímulos são diferentes. Meck e Church demostraram que ratos podem transferir uma representação de estímulo de duração média, originalmente obtida com estímulo luz, para um estímulo sonoro, portanto, de outra modalidade física.

Pavlov (1927) já propunha a existência de uma forma rudimentar de aprendizagem conceitual em animais, que tornaria os animais capazes de responder a atributos básicos de estímulos, independentemente de suas modalidades sensoriais. Gibson (1969) propôs a existência de uma capacidade de informação amodal de atributos básicos de estímulos, que seria responsável pela mediação das funções inter-modais. Meck e Church (1982) comentam que "a habilidade de animais de abstrair atributos temporais de modalidades sensoriais implica que algumas representações internas de estímulo não são rigidamente determinadas pelas características do estímulo externo" (p. 240).

No presente experimento, embora não seja usado um procedimento de manipulação de componentes amodais com 
testes inter-modais, são empregados estímulos que têm a mesma modalidade física luminosa, porém com propriedades excitatórias ou inibitórias adquiridas, ligadas respectivamente a cada uma dentre duas diferentes durações. Pretendeu-se examinar se o controle exercido por estímulos em função de seus componentes amodais se aplica a procedimentos de aprendizagem complexa, tais como o de discriminação condicional. Pretendeu-se verificar se a duração, como uma informação amodal relativa a atributos básicos de estímulos, tem capacidade de adquirir propriedades condicionais excitatórias e inibitórias em animais, em procedimentos de discriminação condicional seriada. Procurou-se verificar se o animal responde diferentemente a estímulos condicionais de característica positiva ou negativa, que têm a mesma modalidade física luminosa, porém com propriedades excitatórias e inibitórias ligadas, respectivamente, a suas diferentes durações.

\section{Método}

\section{Sujeitos}

Foram empregados 16 ratos Wistar, com aproximadamente 90 dias no início do experimento, experimentalmente ingênuos, provenientes do Biotério Central do Campus da Universidade de São Paulo (USP), Ribeirão Preto. Estes animais permaneceram em caixas individuais no biotério do Laboratório de Processos Associativos, Controle temporal e Memória e foram mantidos a $85 \%$ do peso normal através de privação de água.

\section{Equipamento}

O experimento foi realizado em um cubículo revestido com eucatex acústico para isolamento de ruídos externos, onde havia uma estante de madeira com oito caixas experimentais (modelo Lafayete 80201$)$. As caixas (20x20x23cm) possuem paredes laterais e piso (em barras) de aço inoxidável, parede frontal, de fundo e teto de acrílico. O bebedouro localiza-se no centro da parede lateral esquerda. Além da iluminação ambiental (luz vermelha), localizada no teto da estante onde as caixas ficam. Um outro tipo de iluminação foi proporcionado por uma lâmpada de 5 watts $(\mathrm{L})$ normalmente desligada, que era ligada em flashs, conforme o procedimento. Esta lâmpada foi utilizada como estímulo característica $(\mathrm{L})$. Os estímulos auditivos utilizados foram: um tom (T) de $1000 \mathrm{~Hz}$ e $30 \mathrm{~dB}$, produzidos por um gerador de áudio modelo RFM (Departamento de Fisiologia da Faculdade de Medicina de Ribeirão Preto - USP); um ruído branco $(\mathrm{R})$ de $68 \mathrm{~dB}$ produzido por um gerador de ruído modelo 901B (Grason-Stadler; Massachusetts) e um clique (C) produzido originalmente por um metrônomo Mähzel com andamento $\tilde{o}=80 \mathrm{~mm}$. Todos os estímulos auditivos foram liberados através de três alto falantes de $20 \mathrm{~W}$. O controle experimental foi realizado por uma interface desenvolvida no Laboratório de Processos Associativos, Controle Temporal e Memória e um computador PC XT, que acionavam automaticamente os mecanismos de reforço e de estímulos.
Foi empregado um programa para procedimentos de Discriminação Condicional. Uma câmera de televisão foi montada a 2 metros de distância em frente às caixas experimentais de modo que pudesse incluir quatro caixas no seu campo de visão. Outra câmera acoplada à porta filmou as outras quatro caixas restantes. Um gravador de vídeo cassete modelo VR9837ATo 1 (Magnavox, Canadá), em conjunto com o monitor de televisão modelo PB12A4 (Philco, Brasil), foi programado para registrar comportamentos ocorrendo $5 \mathrm{se}-$ gundos antes da apresentação da prática e durante cada prática. Após cada prática, programada de acordo com o experimento, o gravador era desligado.

\section{Procedimento}

Todos os ratos foram, inicialmente, submetidos a duas sessões de treino de ida ao bebedouro com duração de 20 min cada sessão. A água foi liberada independentemente da resposta, em esquema de tempo variável de $1 \mathrm{~min}$, totalizando 20 reforços por sessão.

Foram realizadas, em seguida, 107 sessões diárias de treino de discriminação condicional seriada de característica positiva e negativa. Os animais foram distribuídos aleatoriamente em 2 grupos de 8 ratos cada. As práticas correspondentes ao treino de discriminação condicional de estímulo característica positivo tiveram a seguinte seqüência de eventos: uma luz de $15 \mathrm{seg}$ precedendo um tom de $5 \mathrm{seg}$ seguido de reforço (L15 IV T5+). Em outra prática, um tom $5 \mathrm{seg}$ apresentado sozinho não era seguido de reforço (T5-). Nas práticas de discriminação condicional de estímulo característica negativo, uma luz de 5 seg precedia um ruído de $15 \mathrm{seg}$ que não era seguido de reforço (L5 IV R15-). Outra prática foi um ruído de $15 \mathrm{seg}$ apresentado sozinho era seguido de reforço (R15+). Entre o estímulo característica e o estímulo condicionado houve sempre um intervalo vazio de $5 \mathrm{seg}$.

Durante esta fase, cada tipo de prática foi apresentada três vezes por sessão. A seqüência das práticas dentro da sessão foi aleatória, bem como os intervalos variáveis interpráticas, que tiveram uma média de 6 minutos (valor mínimo de 4 min e o máximo de $8 \mathrm{~min}$ ).

Em seguida às sessões de treino, foram realizadas sessões de teste de transferência. No teste de transferência 1, foram empregados estímulos com os quais o animal já havia tido experiência em sessões anteriores (ruído e tom), embora com propriedades diferentes. No teste de transferência 2 , foi empregado um estímulo novo (clique) como estímulo alvo. As sessões dos testes duraram 70 min cada.

No teste de transferência 1 (2 sessões), os animais foram submetidos à seguinte série de práticas não reforçadas:uma luz de $15 \mathrm{seg}$ precedeu um tom de $5 \mathrm{seg}$ (L15 IV T5-); um tom de $5 \mathrm{seg}$ foi apresentado sozinho (T5-); uma prática nova com uma luz de 5 seg precedendo um tom de 5 seg (L5 IV T5-); uma luz de 5 seg precedeu um ruído de $15 \mathrm{seg}$ (L5 IV R 15-); um ruído de $15 \mathrm{seg}$ foi apresentado sozinho (R15-); uma prática nova com uma luz de $15 \mathrm{seg}$ precedeu um ruído $15 \mathrm{seg}$ (L15 IV R15-). Cada tipo de prática foi apresentada duas vezes por sessão, a seqüência foi aleatória. Os intervalos inter-práticas 
foram randomizados e tiveram a média de duração daqueles das sessões de treino.

Em seguida, foram realizadas três sessões de retreino, com a apresentação de práticas idênticas às do treino.

No teste de transferência 2 (duas sessões), foi apresentada aos animais a seguinte série de práticas não-reforçadas: uma luz de 15 seg precedeu um clique de 5 seg (L15 IV C5-); um clique de $5 \mathrm{seg}$ foi apresentado sozinho (C5-); uma luz de 5 seg precedeu um clique de 15 seg (L5 IV C15-); um clique de $15 \mathrm{seg}$ foi apresentado sozinho (C15-). Cada prática foi apresentada três vezes por sessão, em seqüência aleatória. Os intervalos inter-práticas foram randomizados e tiveram a mesma média de duração daqueles das sessões de treino.

Procedimento de observação comportamental. Todas as sessões foram gravadas em vídeo-tape. Cada comportamento do rato foi observado a intervalos de 1,25 seg durante as práticas, sendo um e somente um comportamento registrado. Foram registradas nove categorias de comportamento a saber:

1. H - head-jerk: movimentos curtos e rápidos da cabeça no sentido horizontal e vertical.

2. J - lamber e/ou farejar o bebedouro com movimentos de cabeça em scanning (mas sem que sejam do tipo headjerk) com o focinho dentro da área retraída do bebedouro.

3. K - farejar próximo ao bebedouro: movimentos de cabeça em scanning, com o focinho na metade da caixa onde está localizado o bebedouro.

4. R - farejar: movimentos de cabeça em scanning, com o focinho na metade da caixa oposta ao bebedouro.

5. T - levantar-se: duas patas anteriores afastadas do chão, mantendo-se sobre as duas patas posteriores, sendo ou não acompanhado de movimentos de cabeça em scanning:

6. M - limpeza: coçar-se, lamber o pêlo ou movimento de limpeza da face.

7. G - parado no bebedouro ou próximo ao bebedouro: permanecer imóvel, com o focinho dentro da área retraída do bebedouro ou na metade da caixa onde está localizado o bebedouro.

8. C - parado: permanecer imóvel, com o focinho na metade oposta ao bebedouro.

9. Q - locomoção: movimentação de uma metade da caixa experimental para a outra ou rotação de $360^{\circ}$.

Análise de dados. A medida de porcentagem total de comportamento foi calculada dividindo-se o número de registros de um comportamento particular pelo número total de observações feitas. As categorias comportamentais head-jerk, lamber e $\backslash$ ou farejar o bebedouro foram agrupadas, para efeito de análise, uma vez que atendem à mesma propriedade funcional, como respostas de orientação ao bebedouro. Da mesma maneira, as categorias comportamentais Locomoção, Levantar-se, Farejar e Farejar próximo ao bebedouro foram agrupadas, para efeito de análise, uma vez que atendem à mesma propriedade funcional, de tipo exploratório. Não foram analisadas categorias com freqüências muito baixas: Parado, Parado no bebedouro, Parado próximo ao bebedouro e Limpeza.

A categoria agrupada de orientação ao bebedouro foi considerada como indicadora da aquisição de desempenho na fase de treino e nos testes. A categoria agrupada de tipo exploratório foi empregada na apresentação dos dados da fase de treino para permitir a descrição das mudanças ocorridas nas outras respostas do repertório comportamental.

A Prova de Wilcoxon para duas amostras relacionadas (Siegel, 1975) foi empregada para análise do desempenho dos animais durante os estímulos alvo nas 5 últimas sessões de treino e nos testes de transferência 1 e 2. Esta análise foi precedida pela Prova de Friedman no Teste 1, uma vez que o Teste 1 envolveu a comparação entre três condições estímulo alvo. Foi usado o nível de significância $p=0,05 \mathrm{em}$ todas as análises.

Foi realizado um teste de concordância entre dois observadores a respeito das categorias comportamentais analisadas, sendo obtido um índice de concordância maior que $90 \%$ das observações.

\section{Resultados}

\section{Treino}

Os dados estão apresentados nas Figuras através das médias dos sujeitos, para facilitar a leitura das tendências, mas a descrição dos resultados considera apenas as diferenças apontadas como significativas pelo tratamento estatístico.

Dados das últimas cinco sessões do treino indicam aquisição de discriminação condicional. Nas práticas de discriminação condicional positiva, as freqüências de respostas de orientação ao bebedouro - head-jerk, lamber e $\backslash$ ou farejar o bebedouro - foram maiores durante o estímulo condicionado precedido pelo estímulo característica positivo (L15 IV)T5+ do que durante o estímulo condicionado apresentado sozinho T5- (Figura 1) (Wilcoxon, $P=0,0006$ ). Nas práticas de discriminação condicional negativa, as freqüências de respostas de orientação ao bebedouro foram

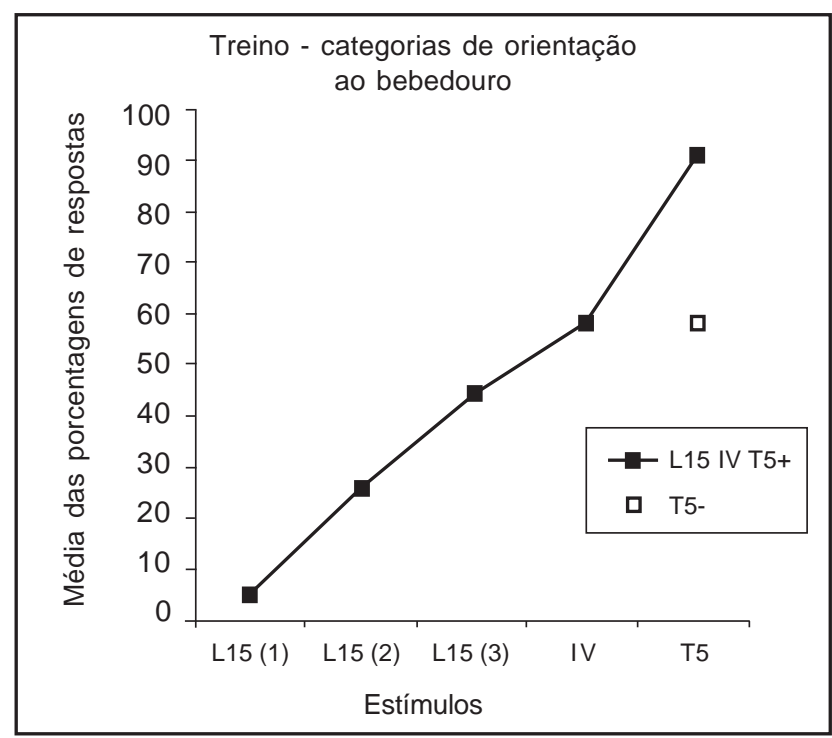

Figura 1. Médias das porcentagens de resposta das categorias de orientação ao bebedouro agrupadas (head-jerk+lamber e $\backslash$ ou farejar o bebedouro) nas práticas L15 IV T5+ e T5-, durante as cinco últimas sessões da fase de aquisição. $\mathrm{L}=$ luz; $\mathrm{T}=\mathrm{Tom}$; $\mathrm{IV}=$ intervalo vazio; L15(1), L15(2) e L15(3) correspondem a blocos de 5 segundos de L 15 . 
menores, a partir do segundo bloco de 5 segundos, durante o estímulo condicionado precedido pelo estímulo característica negativo (L5 IV)R15- do que durante o estímulo condicionado apresentado sozinho R15+ (Figura 2) (Wilcoxon, $P=0,01$ e $P=0,02$ respectivamente).

A distribuição de respostas de orientação ao bebedouro durante a prática composta de estímulo característica positivo ocorreu com um aumento gradativo desde o primeiro bloco de estímulo característica até o estímulo condicionado (Figura 1). Na discriminação condicional negativa, o aumento de respostas no decorrer da prática é semelhante ao de discriminação positiva, porém, com uma desaceleração da curva após o primeiro bloco de estímulo condicionado (Figura 2).

Nas práticas de discriminação condicional positiva, as freqüências de categorias de respostas de tipo exploratório agrupadas (Locomoção, Levantar-se, Farejar e Farejar próximo ao bebedouro) foram menores durante o estímulo condicionado precedido pelo estímulo característica positivo (L15 IV)T5+ do que durante o estímulo alvo apresentado sozinho T5- (Figura 1b) (Wilcoxon, $P=0,001475$ ). Nas práticas de discriminação condicional negativa, as freqüências de categorias de tipo exploratório agrupadas foram maiores, a partir do segundo bloco de 5 segundos, durante o estímulo alvo precedido pelo estímulo característica negativo (L5 IV)R 15- do que durante o estímulo alvo apresentado sozinho R15+ (Figura 2b) (Wilcoxon, $P=0,010$ e $P=0,02$ respectivamente).

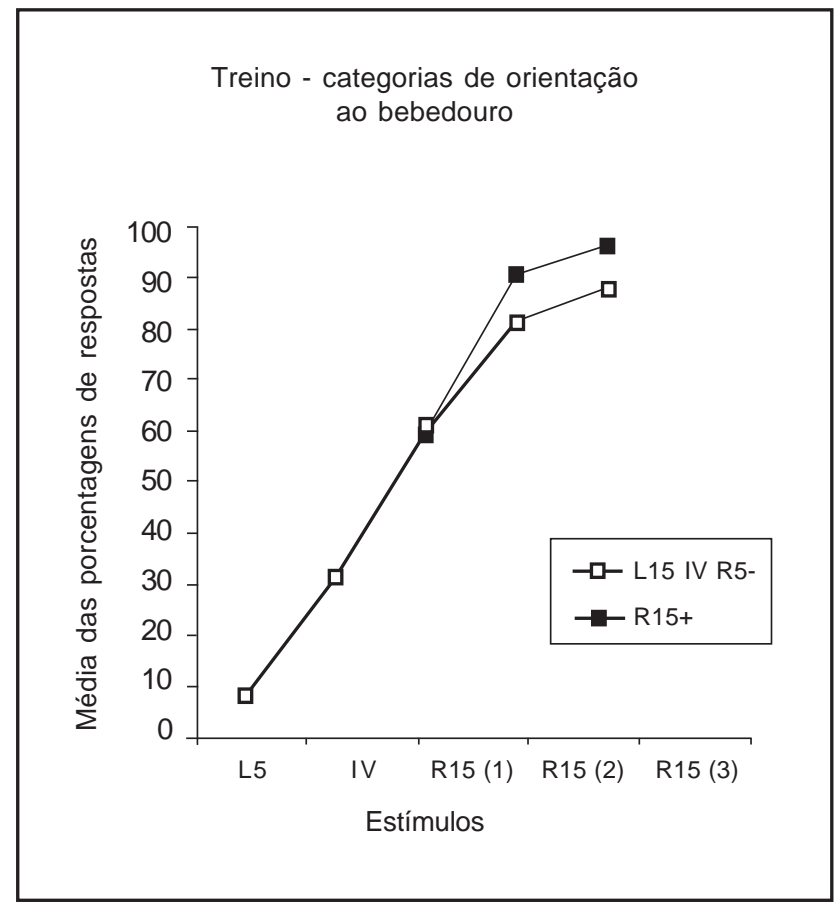

Figura 2. Médias das porcentagens de resposta das categorias de orientação ao bebedouro agrupadas (head-jerk+lamber e $\backslash$ ou farejar o bebedouro) nas práticas L5 IV R15-e R15+ durante as cinco últimas sessões da fase de aquisição. $\mathrm{L}=\mathrm{luz} ; \mathrm{R}=\mathrm{Ruído}$; $\mathrm{IV}=$ intervalo vazio. R15(1), R15(2), R15(3) correspondem a blocos de 5 segundos de R 15 .
A distribuição de categorias de tipo exploratório agrupadas durante a prática composta de estímulo característica positivo apresentou uma diminuição gradativa desde o primeiro bloco de estímulo característica até o estímulo condicionado (Figura 1b). Na discriminação condicional negativa (Figura $2 \mathrm{~b}$ ), ocorreu uma redução de respostas, no decorrer da prática, semelhante à da discriminação condicional positiva, porém, com uma desaceleração de curva após o primeiro bloco do estímulo alvo.

Teste de transferência 1. A Figura 3 apresenta a média da porcentagem de respostas de orientação ao bebedouro durante a apresentação do estímulo alvo T5 precedido ou não pelo estímulo característica de duração de 15 segundos ou de 5 segundos (L1 5 ou L5). Houve diferenças entre as condições estímulo alvo T5 sozinho e T5 precedidos por L5 e L15 (Friedmann, $p=0,003$ ). As taxas de respostas ao estímulo alvo sozinho T5 (treinado sem liberação de reforço) foram menores que as taxas de respostas ao estímulo alvo precedido do estímulo característica L15, replicando os dados já obtidos na fase de treino: T5- $<$ (L15 IV)T5(Wilcoxon, $P=0,0009$ ). As taxas de respostas ao estímulo alvo sozinho T5 também foram menores que as taxas de respostas ao estímulo alvo precedido do estímulo característica negativo L5: T5- $<$ (L5 IV)T5-(Wilcoxon, $P=0$,0006). Porém, as taxas de respostas ao estímulo alvo precedido pelo estímulo característica negativo L5 - prática

Treino - categorias de tipo exploratório

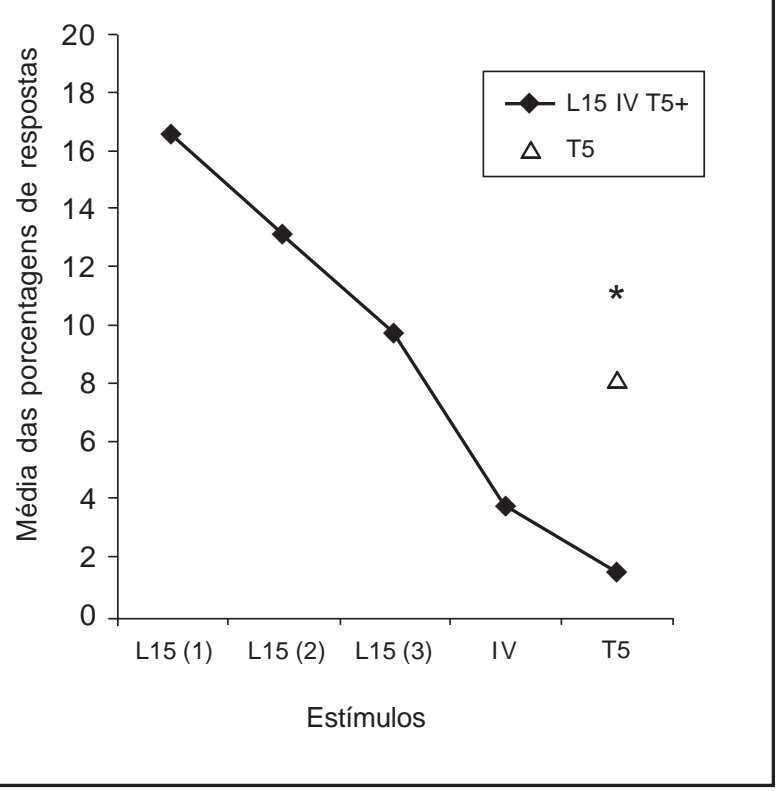

Figura 16 . Médias das porcentagens de resposta das categorias de tipo exploratório agrupadas (locomoção, levantar-se, farejar e farejar próximo ao bebedouro) nas práticas L15 IV T5+ e T5-, durante as 5 últimas sessões da fase de aquisição. $\mathrm{L}=$ luz; $\mathrm{T}=\mathrm{Tom}$; IV = intervalo vazio; L1(15), L2(15) e L3(15) correspondem a blocos de 5 segundos de L 15 . 
Treino - categorias de tipo exploratório

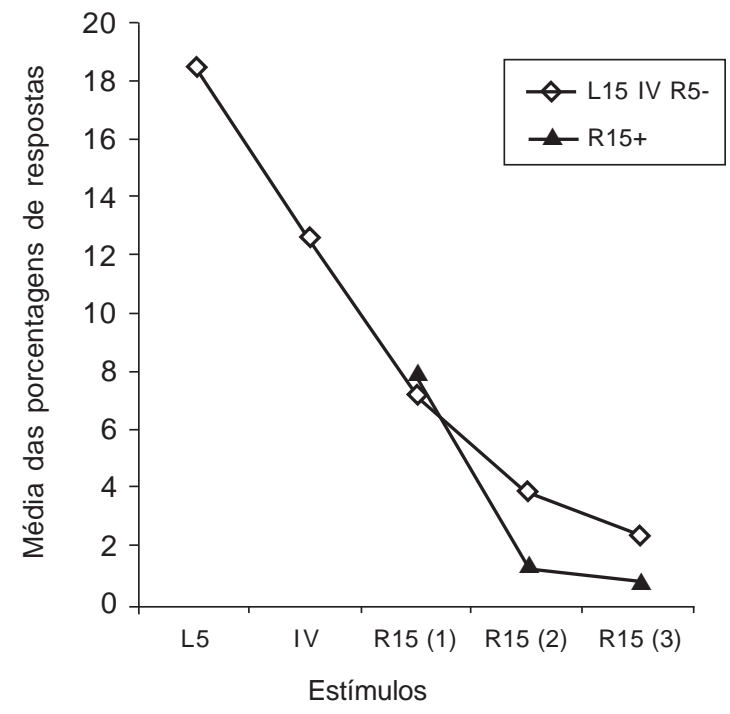

Figura 2b. Média das porcentagens de respostas das categorias de tipo exploratório agrupadas (locomoção, levantar-se, farejar e farejar próximo ao bebedouro) nas práticas L5 IV R15- e R 15+, durante as 5 últimas sessões da fase de aquisição. $\mathrm{L}=$ luz; $\mathrm{R}=$ Ruído; IV= intervalo vazio; R15(1), R15(2) e R15(3) correspondem a blocos de 5 segundos de R 15 .

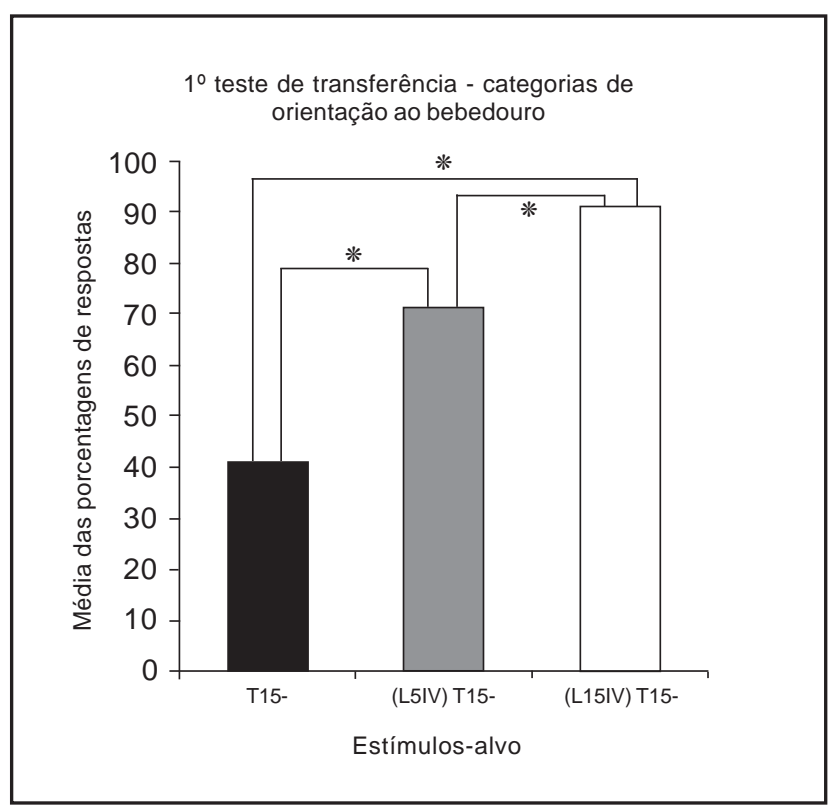

Figura 3. Médias das porcentagens de resposta das categorias de orientação ao bebedouro agrupadas (head-jerk+lamber e \ou farejar o bebedouro) no 10 teste de transferência, durante a apresentação do estímulo alvo das seguintes práticas: T5-, L5 IV T5-e L1 5 IV T5-. L= luz; T=Tom; IV= intervalo vazio. (Durante a fase de aquisição as práticas foram: L15 IV T5+; T5-; L5 IV R15-; R15+). Diferença estatística (*). introduzida nesta fase de teste - foram menores que quando precedido por L15: (L5 IV)T5- < (L15 IV)T5- (Wilcoxon, $P=0,0033)$.

A Figura 4 apresenta a porcentagem de respostas de orientação ao bebedouro durante a apresentação do estímulo alvo R 15 precedido ou não pelo estímulo característica de duração de 15 segundos ou de 5 segundos (L15 ou L5). As taxas de respostas ao estímulo alvo precedido pelo estímulo característica negativo L5 foram menores que as taxas de resposta ao estímulo alvo R 15 sozinho (treinado com liberação de reforço): (L5 IV)R 15- < R15- (Wilcoxon, $P=0,002$ ), replicando os dados obtidos na fase de treino. Não houve diferenças entre as taxas de respostas ao estímulo alvo R 15 sozinho e quando foi precedido pelo estímulo característica L15 (Wilcoxon, $P=0$,2933). Porém, as taxas de respostas ao estímulo alvo precedido por L15 - prática introduzida neste teste - foram maiores que as precedidas por L5 (Wilcoxon, $P=0,038$ ).

Teste de transferência 2. A Figura 5, na próxima página, mostra o desempenho dos animais durante as práticas de estímulo característica positivo. Os dados são semelhantes aos obtidos na fase de treino com o estímulo alvo T5: ocorrência de maiores freqüências de respostas de orientação ao bebedouro durante o estímulo alvo novo (clique) precedido de estímulo característica positivo L 15 do que durante o mesmo estímulo apresentado sozinho: (L15 IV)C5- > C5(Wilcoxon, $P=0,001$ ). Da mesma forma, os dados da Figura 6 mostram a ocorrência de freqüências menores de respostas

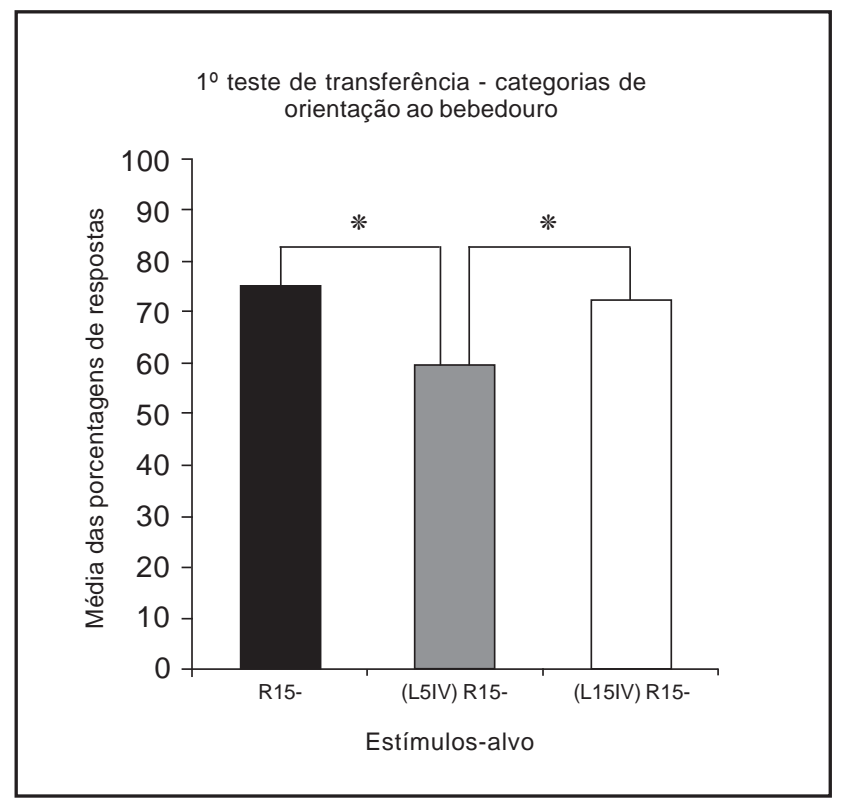

Figura 4. Médias das porcentagens de resposta das categorias de orientação ao bebedouro agrupadas (head-jerk+lamber e \ou farejar o bebedouro) no 10 teste de transferência, durante a apresentação do estímulo alvo das seguintes práticas: R 15-, L15 IV R15- e L5 IV R15-. L= luz; R= ruído; IV= intervalo vazio. (Durante a fase de aquisição as práticas foram: L15 IV T5+; T5; L5 IV R15- ; R15+). Diferença estatística (*). 
durante o estímulo alvo precedido pelo estímulo característica negativo L5 do que durante o mesmo estímulo apresentado sozinho: (L5 IV)C15-> C15-(Wilcoxon, $P=0,0056$ ).

\section{Discussão}

A aquisição de discriminação condicional pelos animais indica que um estímulo luminoso (L), quando apresentado numa duração de 15 segundos em práticas de discriminação condicional positiva, adquiriu a propriedade de estímulo característica positivo; o estímulo de mesma modalidade física luminosa quando apresentado, para o mesmo

$2^{\circ}$ teste de transferência - categorias de orientação ao bebedouro

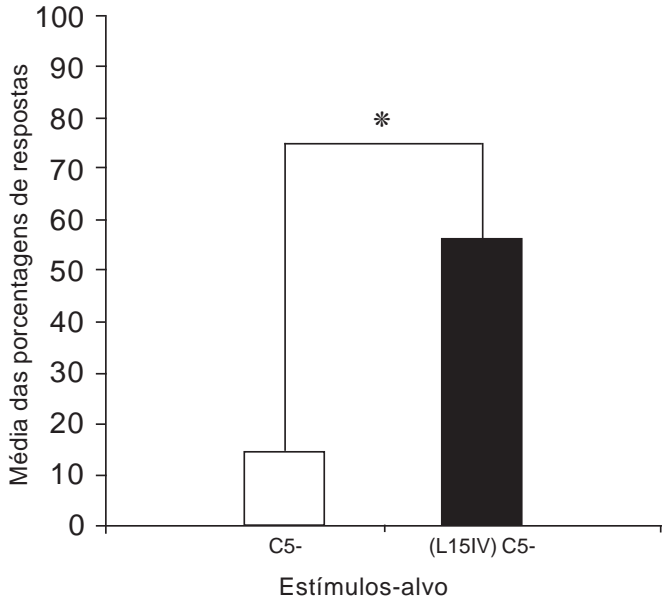

Figura 5. Médias das porcentagens de resposta das categorias agrupadas de orientação ao bebedouro (head-jerk+lamber e $\backslash$ ou farejar o bebedouro) nas práticas L15 IV C5- e C5- no $2^{\circ}$ teste de transferência. $\mathrm{L}=$ luz; $\mathrm{C}=$ Clique; IV= intervalo vazio. (Durante a fase de aquisição as práticas foram : L15 IV T5+; T5-; L5 IV R15-; R15+). Diferença estatística (*).

$2^{\circ}$ teste de transferência - categorias de orientação ao bebedouro

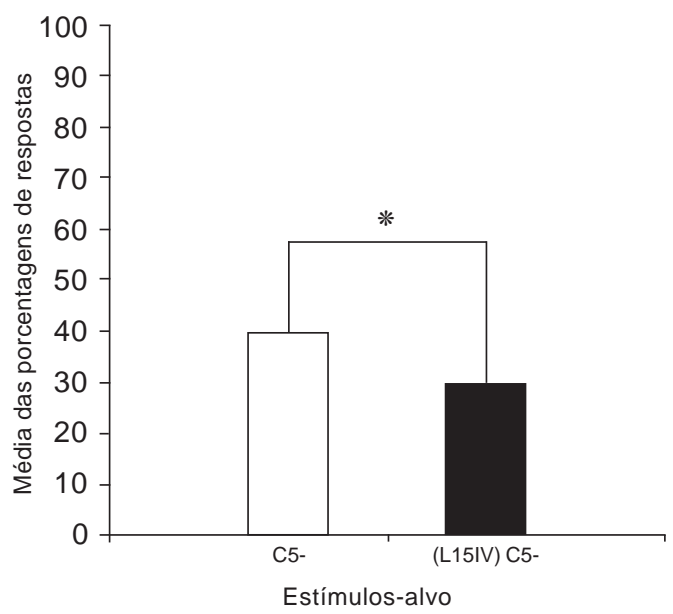

Figura 6. Média das porcentagens de resposta das categorias agrupadas (head-jerk+lamber e $\backslash$ ou farejar o bebedouro) nas práticas L5 IV C15-e C15- no 20 teste de transferência. L= luz; C= Clique; $\mathrm{IV}=$ intervalo vazio. (Durante a fase de aquisição as práticas foram: L15 IV T5+; T5-; L5 IV R15-; R15+). Diferença estatística (*). animal, numa duração mais curta, de 5 segundos, em práticas de discriminação condicional negativa, adquiriu a propriedade de estímulo característica negativo. Assim, estímulos de mesma modalidade física podem adquirir propriedades condicionais distintas, positiva ou negativa, se forem apresentados com durações diferentes em práticas de discriminação condicional positiva e negativa, respectivamente.

Os resultados mostraram que os animais adquiriram a discriminação das propriedades condicionais excitatórias (X) e inibitórias (Y) relativas, respectivamente, a duas durações diferentes de um mesmo estímulo. Embora o procedimento empregado neste estudo não envolva uma manipulação inter-modal (cross-modal), como no estudo de Meck e Church (1982), os resultados confirmam a hipótese de que uma informação amodal relativa a atributos básicos de estímulos, como a duração, pode adquirir propriedades discriminativas com componentes positivos ou negativos do estímulo. Além disso, estes resultados mostram que propriedades mais complexas, como a de um estímulo condicional, podem também ser associadas a representações amodais de estímulo.

A distribuição de respostas durante as práticas sugere que apesar de o animal poder estar sob controle do tempo total de duração da prática, seu repertório comportamental é modulado pelos estímulos presentes no decorrer da prática: aceleração positiva de respostas condicionadas em práticas de discriminação condicional positiva e aceleração negativa em práticas de discriminação condicional negativa.

As diferenças encontradas entre os estímulos condicionados apenas nos dois últimos blocos de 5 seg destes, nas práticas de discriminação condicional negativa, não favorecem uma interpretação em termos de estratégia prospectiva, mas sugerem que o animal pode ter empregado uma estratégia retrospectiva: após o primeiro bloco de estímulo alvo, o animal verificaria na memória de curto prazo a presença ou não do estímulo característica adequado para orientar sua ação.

Os dados do primeiro teste de transferência durante as práticas inter-mixadas indicam não ter havido transferência de propriedades condicionais, uma vez que a apresentação do estímulo de característica negativo L5 não levou os animais a apresentarem taxas de respostas menores que as registradas durante o estímulo alvo T5 sozinho, que já fora treinado com a não liberação de reforço; da mesma forma, a apresentação do estímulo de característica positiva L15 não levou os animais a apresentarem taxas de respostas maiores que as registradas durante o estímulo alvo R 15 sozinho, que já fora treinado com liberação de reforço.

Esta não transferência confirma a hipótese de Holland (1992) de que compostos de estímulos seriais favorecem a aquisição da propriedade do estímulo característica de definir a ocasião em que se dá a associação primária entre estímulo alvo e reforço (occasion setting), o que dificultaria a transferência da propriedade do estímulo característica para estímulos alvo que não estavam envolvidas nesta associação.

Entretanto, embora os dados não permitam o reconhecimento da ocorrência de um efeito de transferência do estí- 
mulo característica negativo, as diferenças entre as respostas registradas durante o estímulo alvo T5 precedido pelo estímulo característica positivo L15 e o negativo L5 indicam que o estímulo característica negativo preservou, nesta prática, algum componente inibitório, uma vez que os animais apresentaram taxas de respostas menores durante o estímulo alvo precedido pelo estímulo característica negativo do que quando precedido pelo estímulo característica positivo. Esta manifestação da propriedade inibitória pode ser entendida em termos de um componente de uma associação de tipo configuracional, presente no estímulo característica. Pearce (1994), examinando dados de procedimento de discriminação condicional simultânea, sugere que o animal resolve tarefas de discriminação condicional por associação configuracional do composto estímulo característicaestímulo alvo com o reforço; a excitação ou inibição associadas ao reforço seriam as únicas variáveis relevantes para a aprendizagem, além das propriedades de generalização dos estímulos. Uma outra possibilidade explicativa para a persistência de um componente inibitório do estímulo característica nesta fase de teste é que, ao mesmo tempo que o desempenho animal esteja sob controle da propriedade de occasion setting do estímulo característica, este também pode estar sob controle da propriedade decorrente da associação primária entre o estímulo característica e o estímulo incondicionado (Rescorla, 1985).

No segundo teste de transferência, a replicação, com o emprego de estímulo alvo novo (clique), das distribuições registradas na fase de treino com estímulos alvo R 15 e T5, pode ser entendida como uma transferência das propriedades condicionais do estímulo característica. Entretanto, os dados obtidos com a apresentação dos estímulos alvo sozinho mostram que a replicação das tendências também ocorre para estes estímulos em práticas nas quais os animais não estão expostos ao estímulo característica. Neste caso, as distribuições encontradas no teste 2 podem ser entendidas, também, como decorrentes da generalização das propriedades físicas do estímulo alvo, uma vez que o estímulo alvo novo é um clique, com propriedades sonoras semelhantes às dos estímulos alvo empregados na fase de treino. Esta possibilidade uma generalização de condições de treino para as de prática com um estímulo alvo novo, sugerida pelos dados, é, também, compatível com uma hipótese de associação configuracional de estímulos em procedimentos de discriminação condicional (Pearce, 1994).

De qualquer forma, os dados deste estudo indicam que o fator temporal pode afetar os processos associativos subjacentes ao comportamento do animal, dependendo das variações com que ele ocorre, ou seja, o animal parece ser capaz de armazenar em sua memória uma representação temporal dos estímulos, e utilizá-la como estratégia de solução para os problemas que seu meio coloca. Esta possibilidade se estende a procedimentos de aprendizagem complexa, incluindo a representação da duração do tempo no imaginário animal (Bueno, 1997).
Bueno, J. L. O. (1997). O imaginário animal. Psicologia USP, 8(2), 165-180.

Bueno, J. L. O., \& Moreira, R. C. (1998). Conditional discrimination: The role of CS-alone trials. Behavioural Processes, 42, 33-45.

Gibson, E. J. (1969). Principles of perceptual learning and development. New York: Appleton-Century-Crofts.

Holland, P. C. (1984). Differential effects of reinforcement of an inhibitory feature after serial and simultaneous feature negative training. Journal of Experimental Psychology: Animal Behavior Process, 10, 461-475.

Holland, P. C. (1985). The nature of conditioned inhibition in serial and simultaneous feature-negative discriminations. In R.R. Miller \& N.E. Spear (Eds.), Information processing in animals: Conditioned inhibition (pp. 267-297). Hillsdale, NJ: Erlbaum.

Holland, P. C. (1992). Occasion setting in Pavlovian conditioning. In D. Medin (Ed.), The psychology of learning and motivation (Vol. 28, pp. 69-125). San Diego, CA: Academic Press.

Holland, P. C., \& Lamarre, J. (1984). Transfer of inhibition after serial and simultaneous feature-negative discrimination training. Learning and Motivation, 15, 219-243.

Jenkins, H. M., \& Sainsbury, R. S. (1969). The development of stimulus control through differential reinforcement. In N. J. Mackintosh \& W. K. Honig (Eds.), Fundamental issues in associative learning (pp. 123-161). Halifax, Canadá: Dalhousie University Press.

Meck, W. H., \& Church, R. M. (1982). Abstraction of temporal attributes. Journal of Experimental Psychology: Animal Behavior Processes, 8, 226-243.

Pavlov, I. P. (1927). Conditioned reflexes. London: Oxford University Press.

Pearce, J. M. (1994). Similarity and discrimination: A selective review and a connectionist model. Psychological Review, 101, 57-607.

Pearce, J. M., \& Hall, G. (1980). A model for Pavlovian learning Variations in the effectiveness of conditioned but not unconditioned stimuli. Psychological Review, 87, 532-552.

Rescorla, R. A. (1985). Inhibition and facilitation. In R. R. Miller $\&$ N. E. Spear (Eds.). Information processing in animals: Conditioned inhibition (pp 299-326). Hillsdale, NJ: Erlbaum.

Rescorla, R. A., \& Holland, P. C. (1977). Associations in Pavlovian conditioned inhibition. Learning and Motivation, 8, 429-447.

Ross, R. T., \& Holland, P. C. (1981). Conditioning of simultaneous and serial feature positive discriminations. Animal Learning and Behavior, 9(3), 293-303.

Siegel, S. (1975). Estatística não-paramétrica para as ciências do comportamento. São Paulo, SP: McGraw-Hill do Brasil.

Wagner, A. R., \& Rescorla, R. A. (1972). Inhibition in Pavlovian conditioning: Applications of a theory. In M. S. Halliday \& R A. Boakes (Eds.), Inhibition and learning (pp 301-336) London: Academic Press.

Recebido: 16/11/2005

$1^{a}$ revisão: $11 / 09 / 2006$

Aceite final: 27/09/2006

\section{Referências}

